\title{
New approaches under development: cardiovascular embryology applied to heart disease
}

\author{
Karl Degenhardt, ${ }^{1}$ Manvendra K. Singh, ${ }^{2}$ and Jonathan A. Epstein ${ }^{2}$
}

\begin{abstract}
1Division of Cardiology, Children's Hospital of Philadelphia, Philadelphia, Pennsylvania, USA. '2Department of Cell and Developmental Biology, Cardiovascular Institute and Institute for Regenerative Medicine, Perelman School of Medicine at the University of Pennsylvania,
\end{abstract} Philadelphia, Pennsylvania, USA.

\begin{abstract}
Despite many innovative advances in cardiology over the past 50 years, heart disease remains a major killer. The steady progress that continues to be made in diagnostics and therapeutics is offset by the cardiovascular consequences of the growing epidemics of obesity and diabetes. Truly innovative approaches on the horizon have been greatly influenced by new insights in cardiovascular development. In particular, research in stem cell biology, the cardiomyocyte lineage, and the interactions of the myocardium and epicardium have opened the door to new approaches for healing the injured heart.
\end{abstract}

\section{Introduction}

The modern era of cardiac surgery began in 1944, when Alfred Blalock and Vivien Thomas combined forces with Helen Taussig to surgically palliate "blue babies" (1). Other significant advances quickly followed, including cardiopulmonary bypass, heart transplantation, mitigation of the risk factors for coronary artery disease (CAD), and catheter-based interventions. Subsequent progress has arguably occurred at a more measured pace.

Nevertheless, recent work in the basic sciences has rapidly advanced our understanding of the molecular and cellular mechanisms that govern cardiovascular biology. In particular, there have been exciting discoveries in the field of cardiac development. These advances are laying the groundwork for the next wave of innovation in cardiovascular medicine and surgery. The potential impact of the study of developmental biology on the understanding of congenital heart disease (CHD) is relatively straightforward. The ramifications for adult cardiology are less intuitive, but innovative physician-researchers are already applying derived knowledge in areas such as stem cell biology to the treatment of adult patients.

$\mathrm{CAD}$ and myocardial infarction remain major causes of heart failure in the adult population. Ischemic injury and myocardial damage resulting from other causes, including toxins or infection, result in loss of functional myocardium and scar formation. New insights into the embryonic origin of myocardium and the molecular underpinnings of myocardial development have led to recent breakthroughs in reprogramming non-muscle cells toward the myocardial lineage, which is a potential approach for regeneration therapy for loss of cardiac muscle due to infarction or disease. Recent studies have begun to elucidate why adult myocardial cells are far less capable of regeneration and cell division than those in the embryonic heart. A better understanding of how embryonic myocytes are instructed to divide and how the adult myocytes of some organisms, such as zebrafish, are able to retain this property may lead to new regenerative approaches.

\section{Heart fields and embryonic myocardial origins}

Classic teaching describes mammalian heart formation in terms of linear heart tube formation, followed by looping, chamber specification, valve formation, and septation. This model posits the spec-

Conflict of interest: The authors have declared that no conflict of interest exists. Citation for this article: J Clin Invest. 2013;123(1):71-74. doi:10.1172/JCI62884. ification of early cardiac myocardial progenitor cells derived from mesoderm that coalesce at the midline to form the linear heart tube around a single layer of endocardial cells. These myocardial precursors were thought to give rise to all of the contractile muscle of the mature heart. Over the past ten years, compelling data from multiple vertebrate organisms have indicated that new myocardium is added to the forming heart at both the arterial and venous poles as well as at stages later than previously recognized $(2,3)$. In the mouse, for example, additional myocardial contributions continue through mid-gestation, long after looping has occurred, and form major portions of the right ventricle, outflow tract, and atria. These relatively late contributions are said to derive from a "second heart field" of mesodermal derivatives characterized by expression of molecular markers distinct from those contributing to the early linear heart tube (the first heart field). The discovery of the second heart field has important implications for our understanding of CHD and the molecular programs that instruct myocardial fate specification and differentiation $(4,5)$.

The critical role of second heart field precursors in cardiac development suggests that some developmental defects relate to abnormal contribution or function of this population of cells (6). For example, hypoplastic right ventricle and some forms of outflow tract defects may result from defective developmental programs specific to second heart field derivatives. Ebstein's anomaly and right ventricular dysplasia are other examples of congenital defects that suggest second heart field associations. Although congenital heart defects are commonly classified on the basis of distinctive structural and phenotypic characteristics, it seems likely that a new classification will emerge, taking into account developmental underpinnings such as those due to specific second heart field defects. Human genetic studies coupled with an increasing understanding of the genetic contributions to development will inform this new classification.

\section{Reprogramming: altering cellular software}

The delineation of the first and second heart fields has aided in our understanding of the molecules, including transcription factors and microRNAs (miRNAs), involved in determining cardiomyocyte lineages. The induction of pluripotency by four transcription factors, OCT3/4, SOX2, KLF4, and MYC opened the possibility of engineering any cell type from a person's own skin or blood cells, with enormous potential for regenerative medicine (7). 
Nevertheless, the ability to efficiently and completely differentiate pluripotent stem cells into cardiomyocytes for functional replacement of injured cardiac muscle remains a challenge $(8,9)$. An alternative approach is to directly convert one differentiated cell type into another - so-called "direct reprogramming." Indeed, the ability of transcription factors to alter the fate of differentiated cells was first established with the conversion of fibroblasts into skeletal muscle cells by MyoD (10), a discovery that long predates the development of induced pluripotent stem cells. Although transdifferentiation by use of a single transcription factor has not been a generalized technique for the production of other cell types, the number of lineages able to be induced by combinations of transcription factors continues to grow. To date, these include macrophages, pancreatic $\beta$-islet cells, brown fat, NKT cells, and neurons (reviewed in ref. 11). Direct reprogramming of cells to cardiomyocytes has been a recent focus of researchers.

Detailed studies of normal cardiac development have identified critical factors expressed at sequential stages of cardiomyocyte specification and lineage restriction. Ieda et al. exploited this knowledge to directly reprogram fibroblasts to cardiomyocytes (12). These investigators reported transdifferentiation of postnatal cardiac and dermal fibroblasts into cardiomyoctyes using the combination of Tbx5, Gata4, and Mef2c. More recent data suggest that these factors may also work in vivo to reduce scarring after infarction $(13,14)$. The efficiency of direct reprogramming with these factors has been challenged (15), and the efficacy of this type of genetic manipulation in humans, as well as its safety, remains to be determined. Nevertheless, direct reprogramming of scar tissue into functional cardiomyocytes remains an attractive approach for regeneration therapy for many reasons. As normal replacement of cardiomyocytes is inefficient at best, utilization of alternative cellular sources, such fibroblasts, may be advantageous. Furthermore, the need to deliver exogenous cells grown ex vivo is avoided, and the risk of teratoma is probably lower than with the use of embryonic or induced pluripotent cells.

miRNAs function differently than transcription factors but share the ability to affect the expression of gene programs and to significantly alter cellular identity (16). A single miRNA can target multiple RNA transcripts encoding proteins that function at multiple steps of the same biological pathway. The overall impact can therefore be quite powerful (17). Moreover, miRNAs offer a potential advantage over transcription factor-based reprogramming strategies, which generally involve the use of viral vectors to deliver factors, in that small molecule mimics or antagonists (antagomirs) of miRNAs can be readily produced and delivered without the potential risks of virus-based therapies (18). Clinical trials in humans to target miRNAs are under way for diseases including hepatitis $\mathrm{C}$. The ability of miRNAs to alter cell fate is underscored by reports that the miR-302/367 cluster can efficiently reprogram somatic cells to pluripotency $(19,20)$. More recently, the combination of miR-1, miR-133, miR-208, and miR-499 has been reported to directly reprogram cardiac fibroblasts to cardiomyocytes (21). The efficiency of reprogramming is greatly enhanced by the concomitant use of a Janus protein tyrosine kinase inhibitor. Administration of this cocktail of miRNAs to ischemic mouse myocardium suggests that reprogramming may also be induced in vivo, though improvement in function has not yet been demonstrated (21).

The ability to more precisely direct the ultimate fate of induced pluripotent cells or of directly reprogrammed tissue likely depends on a thorough understanding of the normal developmental cas- cades that lead to cell fate specification in the heart. The developmental programs that lead to the formation of right ventricular myocytes versus left ventricular myocytes or atrial myocytes or Purkinje cells are distinct, and are likely to be informative for engineering various cardiac derivatives. Hence, the continued delineation of embryonic signals that mediate lineage decisions will continue to inform ongoing attempts to engineer regenerative cardiac tissues.

\section{Endogenous cardiac regeneration: a skill lost with age}

Although injured skeletal muscle can rapidly regenerate, the same is not true for the human heart, which has extremely limited regenerative capacity (8). Other organisms, such as fish and amphibians, display far greater regenerative capabilities for many organs and tissues than is evident in humans. The zebrafish heart, for example, can fully regenerate after surgical removal of as much as $20 \%$ of the ventricle. Cardiac regeneration in fish is achieved by activation, dedifferentiation, and proliferation of mature cardiomyocytes (22). Essentially, complete recovery can also occur after surface cryoinjury or genetic ablation of over $60 \%$ of cardiomyocytes (22-26). These findings have prompted researchers to explore the capacity for cardiac regeneration in mammals and to question whether regenerative capacity lost during evolution could be therapeutically reawakened.

Although many adult human tissues, including liver, skin, and intestine, have significant regenerative capacity, the heart had been thought to be incapable of replacing lost cells until very recently. New evidence suggests that cardiomyocytes may renew themselves at a slow rate, with approximately $1 \%$ turning over annually at the age of 25 years, and $0.45 \%$ at the age of 75 years (27). Recently, Porrello et al. demonstrated that neonatal murine hearts can also regenerate (28). The one-day-old mouse has a capacity to regenerate myocardium after apical resection that is lost by 7 days of age. Using fate mapping with an inducible cardiomyocyte-specific Cre (MerCreMer, under the control of the $\alpha$-myosin heavy chain promoter), Porrello et al. showed that the majority of newly formed cardiomyocytes within the regenerated ventricle are derived from preexisting cardiomyocytes (28). However, possible contribution from stem or progenitor cells was not ruled out. In both the mouse and zebrafish, activation of the epicardium appears to be a key component of the regeneration process, just as the epicardium is critical to normal myocardial development in the embryo $(28,29)$.

Important questions remain with regard to which molecular pathways are activated during murine neonatal cardiac regeneration and why the robust regenerative response is lost with age (30, 31). It remains unclear whether the limited regenerative capacity of the adult mammalian heart represents a limited version of the type of regenerations observed in neonates (which show similarities to what has been observed in zebrafish) or whether the mechanisms are distinct. For example, neonatal regeneration involves proliferation of pre-existing cardiomyocytes, while adult regeneration may involve activation of a resident stem cell population. Elucidation of similarities and differences will help to determine how informative a detailed analysis of zebrafish cardiac regeneration will be to the adult human condition. Pharmacologic or cell-based strategies to favor regeneration over fibrosis after infarct would have significant consequences, even if the effects are only partial. Moreover, strategies and timing for surgical palliation of CHD may require reconsideration if age-limited fetal or neonatal cardiac regeneration in humans is similar to that seen in mice. Perhaps earlier interventions would lead to better results. Indeed, fetal surgery for a growing number of conditions, such as myelomeningocele, yields superior 
A

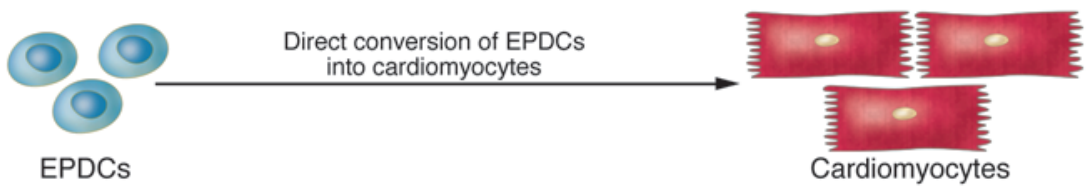

B

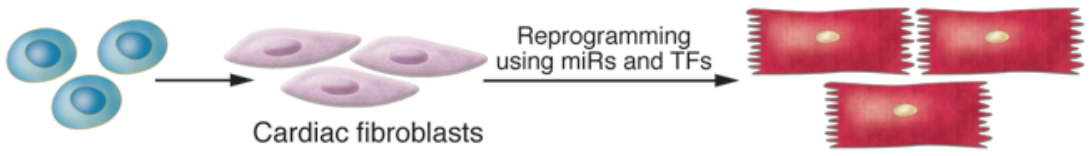

C

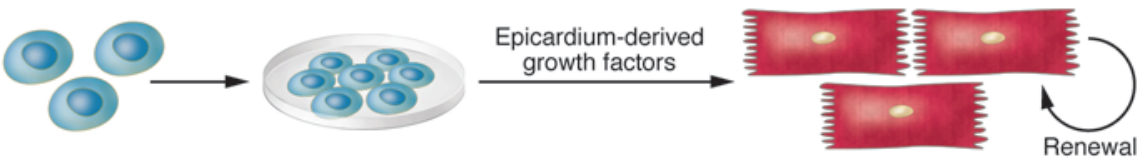

\section{Figure 1}

The normal roles that epicardium and epicardial-derived cells (EPDCs) play in development may have important implications for therapeutic approaches to adult heart disease. (A) Direct conversion of EPDCs to cardiomyocytes appears to occur rarely in development but may be induced by thymosin- $\beta 4$ after injury. (B) Cardiac fibroblasts derive from embryonic epicardium and can be induced to transdifferentiate into cardiac muscle by transcription factors (TFs) or miRNAs. (C) The epicardium is a necessary source of growth factors for normal development of the myocardium. EPDCs may also produce growth factors that could be used therapeutically to enhance cardiomyocyte regeneration. (D) CCFU-Fs are a population of epicardial-derived cells within the heart that have the ability to differentiate to a number of cell types in vitro, including cardiomyocytes.

D

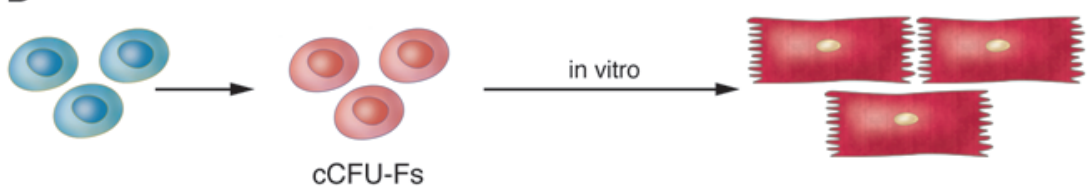

outcomes for the child than does postnatal surgery (32). Fetal interventions for $\mathrm{CHD}$, however, have had limited success to date (33).

\section{Epicardium: an unexpected ally for injured myocardium}

The epicardium is derived from a transient structure called the proepicardial organ (PEO), a cluster of mesothelial progenitor cells located at the venous pole of the heart tube. Soon after it forms, the cells of the PEO migrate onto the surface of the looping heart to envelop the myocardium. Some of these cells then undergo epicardial-to-mesenchymal transition and invade the underlying myocardium, where they differentiate into multiple cell types, while others remain on the surface of the heart and form the epicardium (4, 34-37). The epicardium has classically been considered a passive fibrous lining surrounding the myocardium. However, work from multiple laboratories over the last 10 years has established an active role for epicardium in development, disease, and regeneration.

The PEO is derived from Nkx2.5-expressing and Islet 1 -expressing secondary heart field precursors $(37,38)$. Although the cells composing the PEO appear morphologically similar, they are molecularly heterogeneous. Subcompartments, as revealed by expression of Sema3D, Scx, Wt1, and/or Tbx18, have distinct differentiation potential with regard to the ability to contribute to various cardiac cell types (34). In particular, Sema3D-, Scx-positive proepicardial progenitor cells have the potential to differentiate into coronary endothelial cells (34). Other intracardiac cells that arise from the PEO include fibroblasts, coronary smooth muscle cells, and possibly cardiomyocytes.

The potential of epicardial cells to differentiate into cardiomyocytes could have significant clinical impact because of their relative accessibility (Figure 1). However, the ability of epicardium to contribute to myocardium is controversial. Presently available data suggest that, at best, the myocardial potential of epicardium under normal conditions is low $(35,37,39)$. However, Smart et al. recently attempted to improve epicardial contribution toward cardimyocyte fate by treatment with thymosin- $\beta 4$ before myocardial infarction in rodents (40). Thymosin- $\beta 4$ priming resulted in a significant conversion of epicardial-derived cells into cardiomyocytes after myocardial infarction, accompanied by improved cardiac function (40). This finding is tantalizing, especially considering the translational potential and clinical relevance, but there are conflicting reports and further investigation is required $(41,42)$.

Additional lines of research have also implicated the epicardium as a potential source of myocardial progenitor cells. In a recent report, Chong et al. have identified a new population of cardiac resident cells called "cardiac CFU fibroblasts" (cCFU-Fs) (43). Fatemapping analysis showed that these progenitor cells are derived from epicardium and not from bone marrow, neural crest, or myocardium. In addition to other lineages, cCFU-Fs are also able to differentiate into mesodermal derivatives (smooth muscle cells, cardiac muscle, and adipose tissue) in vitro (43). It remains to be seen whether cCFU-Fs can further differentiate into mature, beating cardiomyocytes either in vitro or in vivo (44).

The epicardium may play an important role after myocardial injury unrelated to any potential for directly contributing to regenerative cardiomyocytes. Myocardial injury of the adult murine heart leads to reactivation of normally quiescent epicardial cells (45). The reactivated epicardial cells re-express embryonic genes and also express markers of fibroblasts and smooth muscle cells (45). Moreover, activated epicardial cells promote angiogenesis through the secretion of paracrine factors. Indeed, conditioned media from these cells reduced infarct size and improved function in an infarction model (45). A variety of paracrine factors secreted from activated epicardium may promote myocardial regeneration in zebrafish and neonatal murine injury models (40, 46, 47). During embryonic development, epicardium and epicardial-derived cardiac fibroblasts regulate myocyte proliferation and ventricular chamber formation 
through pathways that include $\beta 1$-integrin, FGF, and retinoic acid signaling (47-52). Whether cardiac fibroblasts maintain a similar role in the adult heart remains to be fully elucidated.

Notably, most of the efforts to reprogram cells to the cardiomyocyte lineage utilize cardiac fibroblasts as one of the starting materials $(12,13,21)$, and cardiac fibroblasts may be more easily reprogrammed to the cardiomyocyte lineage than non-cardiac fibroblasts. Two reasons for this bias may be postulated based on developmental principles. First, cardiomyocytes and cardiac fibroblasts may be more closely related at an epigenetic level, given a shared precursor population $(37,38)$. Second, cardiac fibroblasts may produce growth factors that support proliferation and differentiation of cardiomyocytes, as occurs in development.

\section{Outlook}

The daunting challenge of understanding the molecular and cellular biology of the developing heart continues to be a productive under- taking that informs therapeutic innovation for adult and pediatric diseases. New insights into the origins of cardiac progenitor cells as well as the molecular pathways governing progressive lineage restriction and functional differentiation of these cells have formed the foundation for exciting regenerative approaches on the horizon.

\section{Acknowledgments}

The authors thank Kurt Engleka for critical reading of the manuscript. This work was supported by grants from the NIH National Heart, Lung and Blood Institute (K08HL094763-02 to K. Degenhardt and UO1 HL100405 to J.A. Epstein), the Spain Fund, and the W.W. Smith Endowed Chair (to J.A. Epstein).

Address correspondence to: Jonathan A. Epstein, 1154 BRB II, 421 Curie Blvd., Philadelphia, Pennsylvania 19104, USA. Phone: 215.898.8731; Fax: 215.573.2094; E-mail: epsteinj@mail.med. upenn.edu.
1. Blalock A, Taussig HB. The surgical treatment of malformations of the heart in which there is pulmonary stenosis or pulmonary atresia. JAMA. 1945;128(3):189-202.

2. Kelly RG, Brown NA, Buckingham ME. The arterial pole of the mouse heart forms from Fgf10 expressing cells in pharyngeal mesoderm. Dev Cell. 2001;1(3):435-440.

3. Cai CL, et al. Isl1 identifies a cardiac progenitor population that proliferates prior to differentiation and contributes a majority of cells to the heart. Dev Cell. 2003;5(6):877-889.

4. Epstein JA. Franklin H. Epstein Lecture. Cardiac development and implications for heart disease. NEngl J Med. 2010;363(17):1638-1647.

5. Parmacek MS, Epstein JA. Cardiomyocyte renewal. NEngl J Med. 2009;361(1):86-88.

6. Gruber PJ, Epstein JA. Development gone awry: congenital heart disease. Circ Res. 2004;94(3):273-283.

7. Takahashi K, Yamanaka S. Induction of pluripotent stem cells from mouse embryonic and adult fibroblast cultures by defined factors. Cell. 2006;126(4):663-676

8. Laflamme MA, Murry CE. Heart regeneration. Nature. 2011;473(7347):326-335.

9. Vieira JM, Riley PR. Chemical genetics and its potential in cardiac stem cell therapy [published online ahead of print March 2, 2012]. BrJ Pharmacol. doi:10.1111/j.1476-5381.2012.01928.x.

10. Davis RL, Weintraub H, Lassar AB. Expression of a single transfected cDNA converts fibroblasts to myoblasts. Cell. 1987;51(6):987-1000.

11. GrafT. Historical origins of transdifferentiation and reprogramming. Cell Stem Cell. 2011;9(6):504-516.

12. Ieda $M$, et al. Direct reprogramming of fibroblasts into functional cardiomyocytes by defined factors. Cell. 2011;142(3):375-386.

13. Qian $\mathrm{L}$, et al. In vivo reprogramming of murine cardiac fibroblasts into induced cardiomyocytes. Nature. 2012;485(7400):593-598.

14. Song $\mathrm{K}$, et al. Heart repair by reprogramming non-myocytes with cardiac transcription factors. Nature. 2012;485(7400):599-604.

15. Chen JX, et al. Inefficient reprogramming of fibroblasts into cardiomyocytes using Gata4, Mef2c, and Tbx5. Circ Res. 2012;111(1):50-55.

16. Boettger T, Braun T. A new level of complexity: the role of microRNAs in cardiovascular development. Circ Res. 2012;110(7):1000-1013.

17. Small EM, Olson EN. Pervasive roles of microRNAs in cardiovascular biology. Nature. 2011; 469(7330):336-342.

18. Obad S, et al. Silencing of microRNA families by seedtargeting tiny LNAs. Nat Genet. 2011;43(4):371-378.

19. Anokye-Danso F, et al. Highly efficient miRNA-medi- ated reprogramming of mouse and human somatic cells to pluripotency. Cell Stem Cell. 2011;8(4):376-388.

20. Miyoshi N, et al. Reprogramming of mouse and human cells to pluripotency using mature microRNAs. Cell Stem Cell. 2011;8(6):633-638.

21. Jayawardena TM, et al. MicroRNA-mediated in vitro and in vivo direct reprogramming of cardiac fibroblasts to cardiomyocytes. Circ Res. 2012; 110(11):1465-1473

22. Poss KD, Wilson LG, Keating MT. Heart regeneration in zebrafish. Science. 2002;298(5601):2188-2190.

23. Chablais F, Veit J, Rainer G, Jazwinska A. The zebrafish heart regenerates after cryoinjury-induced myocardial infarction. BMC Dev Biol. 2011;11:21.

24. Gonzalez-Rosa JM, Martin V, Peralta M, Torres M, Mercader N. Extensive scar formation and regression during heart regeneration after cryoinjury in zebrafish. Development. 2011;138(9):1663-1674.

25. Schnabel K, Wu CC, Kurth T, Weidinger G. Regeneration of cryoinjury induced necrotic heart lesions in zebrafish is associated with epicardial activation and cardiomyocyte proliferation. PLoS One. 2011;6(4):e18503.

26 . Wang J, et al. The regenerative capacity of zebrafish reverses cardiac failure caused by genetic cardiomyocyte depletion. Development. 2011; 138(16):3421-3430.

27. Bergmann O, et al. Evidence for cardiomyocyte renewal in humans. Science. 2009;324(5923):98-102.

28. Porrello ER, et al. Transient regenerative potential of the neonatal mouse heart. Science. 2011; 331(6020):1078-1080.

29. Lepilina A, et al. A dynamic epicardial injury response supports progenitor cell activity during zebrafish heart regeneration. Cell. 2006;127(3):607-619.

30. Li D, Epstein JA. Micro-managing myocyte mitosis. Circ Res. 2011;109(6):611-613.

31. Porrello ER, et al. MiR-15 family regulates postnatal mitotic arrest of cardiomyocytes. Circ Res. 2011;109(6):670-679.

32. Adzick NS, Thom EA, Spong CY, Brock JW. A randomized trial of prenatal versus postnatal repair of myelomeningocele. $N$ Engl J Med. 2011; 364(11):993-1004.

33. Pavlovic M, Acharya G, Huhta JC. Controversies of fetal cardiac intervention. Early Hum Dev. 2008. 84(3):149-153

34. Katz TC, et al. Distinct compartments of the proepicardial organ give rise to coronary vascular endothelial cells. Dev Cell. 2012;22(3):639-650.

35. Cai CL, et al. A myocardial lineage derives from Tbx 18 epicardial cells. Nature. 2008;454(7200):104-108

36. Mikawa T, Gourdie RG. Pericardial mesoderm generates a population of coronary smooth muscle cells migrating into the heart along with ingrowth of the epicardial organ. Dev Biol. 1996;174(2):221-232.

37. Zhou B, et al. Epicardial progenitors contribute to the cardiomyocyte lineage in the developing heart. Nature. 2008;454(7200):109-113.

38. van Wijk B, et al. Epicardium and myocardium separate from a common precursor pool by crosstalk between bone morphogenetic protein- and fibroblast growth factor-signaling pathways. Circ Res. 2009; 105(5):431-441.

39. Christoffels VM, Grieskamp T, Norden J, Mommersteeg MT, Rudat C, Kispert A. Tbx18 and the fate of epicardial progenitors. Nature. 2009;458(7240):E8-E9.

40. Smart N, et al. De novo cardiomyocytes from within the activated adult heart after injury. Nature. 2011;474(7353):640-644

41. Zhou B, et al. Thymosin beta 4 treatment after myocardial infarction does not reprogram epicardial cells into cardiomyocytes. J Mol Cell Cardiol. 2012;52(1):43-47.

42. Kispert A. No muscle for a damaged heart: thymosin beta 4 treatment after myocardial infarction does not induce myocardial differentiation of epicardial cells. J Mol Cell Cardiol. 2012;52(1):10-12.

43. Chong JJ, et al. Adult cardiac-resident MSC-like stem cells with a proepicardial origin. Cell Stem Cell. 2011;9(6):527-540

44. Singh MK, Epstein JA. Epicardium-derived cardiac mesenchymal stem cells: expanding the outer limit of heart repair. Circ Res. 2012;110(7):904-906.

45. Zhou B, et al. Adult mouse epicardium modulates myocardial injury by secreting paracrine factors. J Clin Invest. 2011;121(5):1894-1904.

46. Duan J, et al. Wnt1/betacatenin injury response activates the epicardium and cardiac fibroblasts to promote cardiac repair. EMBOJ. 2012;31(2):429-442.

47. Kikuchi K, et al. Retinoic acid production by endocardium and epicardium is an injury response essential for zebrafish heart regeneration. Dev Cell. 2011;20(3):397-404.

48. Ieda $\mathrm{M}$, et al. Cardiac fibroblasts regulate myocardial proliferation through beta1 integrin signaling. Dev Cell. 2009;16(2):233-244.

49. Chen TH, et al. Epicardial induction of fetal cardiomyocyte proliferation via a retinoic acid-inducible trophic factor. Dev Biol. 2002;250(1):198-207.

50. Guadix JA, et al. Wt1 controls retinoic acid signalling in embryonic epicardium through transcriptional activation of Raldh2. Development. 2011; 138(6):1093-1097.

51. Lavine KJ, et al. Endocardial and epicardial derived FGF signals regulate myocardial proliferation and differentiation in vivo. Dev Cell. 2005;8(1):85-95.

52. Tian Y, Morrisey EE. Importance of myocyte-nonmyocyte interactions in cardiac development and disease. Circ Res. 2012;110(7):1023-1034. 